\title{
Wheel Bearing Fault Isolation and Prognosis Using Acoustic Based Approach
}

\author{
Jianshe Feng ${ }^{1}$, Xinyu $\mathrm{Du}^{2}$, and Mutasim Salman ${ }^{3}$ \\ ${ }^{1}$ NSF I/UCR Center for Intelligent Maintenance Systems, Department of Mechanical Engineering, University of Cincinnati, \\ PO Box 210072, Cincinnati, Ohio 45221-0072, USA. \\ js.feng1990@gmail.com \\ ${ }^{2,3}$ General Motors Research \& Development, Warren, MI, 48092, USA \\ xinyu.du@gm.com \\ masalman53@gmail.com
}

\begin{abstract}
Wheel bearing fault detection, isolation and failure prognosis are critical to improve perceived quality and customer experience for retail vehicles, and to reduce the repair cost and down time for fleet vehicles. Currently, most of the research in bearing failure and degradation diagnosis focus on vibration signal analytics. However, these techniques are rarely applied in automotive industry due to the high sensor cost, installation space limitation, and limited communication bandwidth. In this work, an acoustic based approach for wheel bearing fault detection and isolation is developed to overcome these limitations. Since the bearing noise is a precursor of bearing failure, the proposed method is a prognosis solution. The whole solution is verified using the data collected from a production vehicle. The results show that the proposed method can predict the wheel bearing failure with promising accuracy and robustness.
\end{abstract}

\section{INTRODUCTION}

The vehicle wheel bearing is one of the critical components of vehicles chassis. The bearing failure may cause severe safety issues that involve fateful consequences. Bearing faults refer to deterioration or defects of the bearing components including outer race, inner race, rolling element and cages (McInerny \& Dai, 2003). Typical root causes of failures include excessive external force, bad engineering design, degradation and wear out, and corrosion. Fault diagnosis can play an important role to ensure the safety and availability of the vehicles (Zhang \& Du, 2014).

\footnotetext{
Jianshe Feng et al. This is an open-access article distributed under the terms of the Creative Commons Attribution 3.0 United States License, which permits unrestricted use, distribution, and reproduction in any medium, provided the original author and source are credited.
}

Considering that the wheel bearing defects can impact vehicle driving, it is natural to check some vehicle signals if deviate from their nominal values to identify the wheel bearing defects. These signals include wheel speed fluctuation, bearing temperature rising, vehicle efficiency decreasing, etc. However, the wheel speed fluctuation and vehicle efficiency decreasing are impacted by various internal and external factors. It's very challenging to be employed as a fault signature to identify vehicle bearing faults. Though the wheel bearing temperature can be a useful indicator to reflect the health condition of bearing, there is no thermal couple mounted closed to wheel bearing for most production vehicles. In summary, it is not feasible to find out a good fault indicator from the existing vehicle signals

Besides the existing signals in the vehicle, another approach is to develop the wheel bearing diagnosis techniques using external signals from the add-on sensors. In the history of the bearing diagnosis and prognosis development, the sensor signals (such as vibration, temperature, noise, etc.) related approaches have been investigated extensively (Randall \& Antoni, 2011). The methods adopted to analyze bearing vibration signals include time domain analysis and frequency domain analysis. For time domain analysis, typical approaches are statistical analysis and probabilistic analysis. Some statistics are computed based on the time domain signals and compared with pre-defined references to evaluate the bearing health conditions (Prieto, Cirrincione, Espinosa, Ortega, \& Henao., 2013). For frequency domain analysis, researchers usually focus on the vibration signal collected from accelerometer. Digital signal processing (DSP) techniques such as Fourier transform (Rai \& Mohanty, 2007), envelope analysis (Randall \& Antoni, 2001), Empirical Mode Decomposition (EMD) (Yu, Cheng, \& Yang, 2005) and wavelet transform (Jing \& Qu, 2000) (Kankar, Sharma, \& Harsha, 2011) are used to design fault signatures to indicate 
the bearing degradation or failure. However, the vibration signals are not always available in a commercial product.

Both time domain analysis and frequency domain analysis are aimed at finding out the signatures to distinguish the defected bearing from a healthy one. Usually, the signatures are specially designed based on the investigation of system characteristics with domain knowledge. Once a promising signature is developed or discovered, the diagnosis can be done easily based on rules. However, sometimes it is difficult to discover a useful signature. Recently, some machine learning methods such as neural networks (Li, Chow, Tipsuwan, \& Hung., 2000) and support vector machine (SVM) (Yang, Zhang, \& Zhu, 2007) are also applied to build a classifier after being trained using historical data or features to differentiate whether the bearing condition is healthy or not. These approaches are effective especially when there are no promising fault signatures developed manually. One issue of these data-driven methods is that it is not interpretative compared with physics-model based methods.

In the field, the experienced drivers or technicians diagnose the bearing faults based on the abnormal noise during driving. This inspires us to study the wheel bearing diagnosis and prognosis using acoustic signals. The feasibility of applying acoustic emission (AE) to bearing failures detection was studied by Tan (Tan, 1990). It is found that AE activity increases exponentially with defect size and rotation speed. Zhang et al proposed a bearing defect detection method based on time-frequency analysis by using trackside acoustic signal (Zhang, Lu, He, \& Kong, 2016). The limitation of these studies is that the studies are still in the lab environment, and therefore, the robustness and accuracy in real applications need further validation.

Currently, the acoustic-based approach is not as well developed as vibration signal-based analysis. Few studies can be found related to vehicle wheel bearing diagnosis and prognosis using acoustic signals. To fill this gap, the objective of this paper is to develop, evaluate, and verify an acoustic-based wheel bearing diagnosis and isolation approach. This proposed method can be implemented onboard to enhance the on-board integrated vehicle diagnosis and prognosis performance. The proposed acoustic signalbased wheel bearing fault diagnosis approach is described in the next section in detail, followed by the validation and comparison. The conclusion and future work are given at the end of the paper.

\section{Acoustic-based Wheel Bearing Fault Diagnosis}

In this section, the acoustic signal-based wheel bearing fault diagnosis approach is described in detail including the selection and extraction of fault signatures, and the decisionmaking logic. The proposed approach is outlined in Figure 1. The input is the acoustic signals collected from microphones mounted in the vehicle. Firstly, the time-frequency analysis is employed to generate frequency response of the acoustic signal. Based on the initial outcome, some operations such as averaging and residual calculation are applied to enhance the frequency analysis results. Some useful features, which are related to the health condition of the wheel bearings, are then extracted and selected to reduce the data dimension and computational cost. Thereafter, a single dataset-based wheel bearing fault detection (FD) module is developed. Based on it, the wheel bearing FD algorithms is established.

The acoustic signals collected in this work are from 2 microphones mounted on the front dashboard (denoted as microphone F) and inside the rear trunk (denoted as microphone R) with a $2000 \mathrm{~Hz}$ sampling rate. For frequency analysis, the wheel speed signals are collected from the vehicle CAN bus with a $100 \mathrm{~Hz}$ sampling rate. Figure 2 shows two acoustic signal examples collected from microphone $\mathrm{F}$ for (a) a healthy wheel bearing and (b) a faulty wheel bearing. From the time domain, it is difficult to find out the difference. The wheel speed signal collected from the vehicle can be converted to the frequency domain in the unit of Hertz with the tire diameter information. A DC bias is also noticed in the data which may be inherent for different microphones. The diagnostics approach is expected to be robust again the bias.

To capture the characteristic of the audio signal in both time and frequency domains simultaneously, short time Fourier transform (STFT) is applied to the raw audio signals. The parameters of STFT are set as follows: the window duration is 1 second, the window sliding overlap rate is 0.5 second, the frequency resolution $\Delta f=1 \mathrm{~Hz}$, and the interested frequency range is $[0,500] \mathrm{Hz}$.

Considering the physics of bearing, the frequency response of the audio signals remains time invariant if the vehicle speed is constant. Therefore, for a short period that the vehicle speed is constant, we can remove the noise by

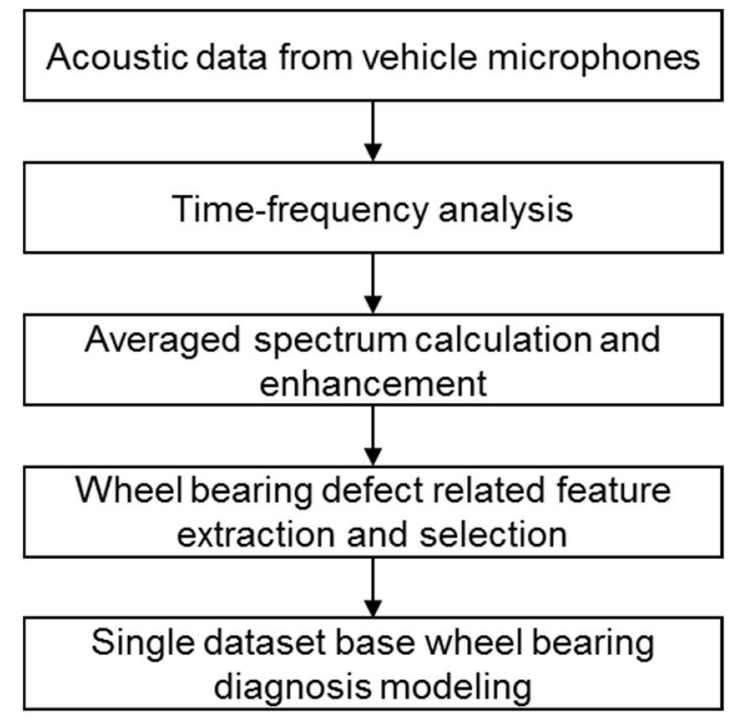

Figure 1 Overall flowchart of acoustic-based wheel bearing fault diagnosis approach 


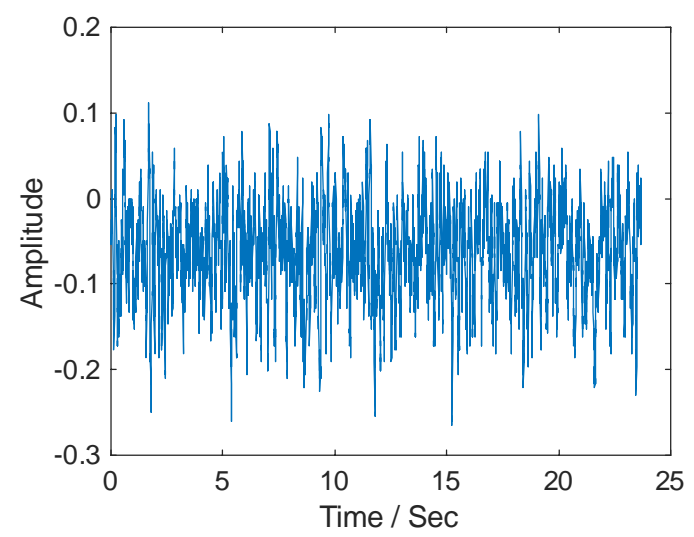

(a)

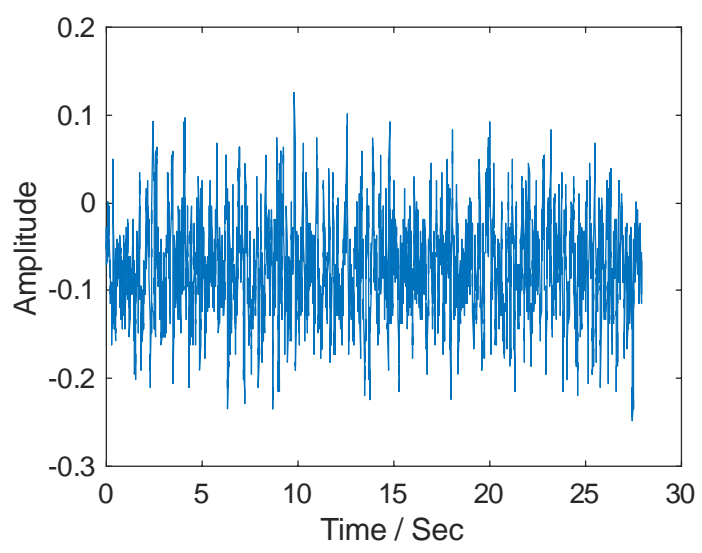

(b)

Figure 2. The acoustic signals in time domain from the microphone F with (a) healthy wheel bearing and (b) faulty wheel bearing

averaging the spectrum in time direction, as shown in Figure 3. After spectrum averaging, the noise information in the spectrum reduces, and consequently, the signal noise ratio (SNR) increases, which improves the spectrum quality.

The comparison of averaged spectrum between a "good" bearing and a "bad" bearing is shown in Figure 3. We can find out that there are some peaks at 12 times (denoted as $12 \mathrm{X}), 18$ times (18X) and 24 times (24X) of fundamental frequency (wheel rotating speed) for a faulty wheel bearing. However, for the healthy wheel bearing, the harmonics phenomena don't exist. Please note that the slow peak at 350 $\mathrm{Hz}$ in Figure 3(a) is not related to the bearing noise based on its shape and frequency. It doesn't show up in other test data. It corresponds to some other noises collected during the test. Please note that the 12X, 18X and 24X frequencies are not a function of the outer race rate but it may be related to bearing geometry, the vehicle system and the media that the acoustic sound travels. Further investigation will be done in the future.

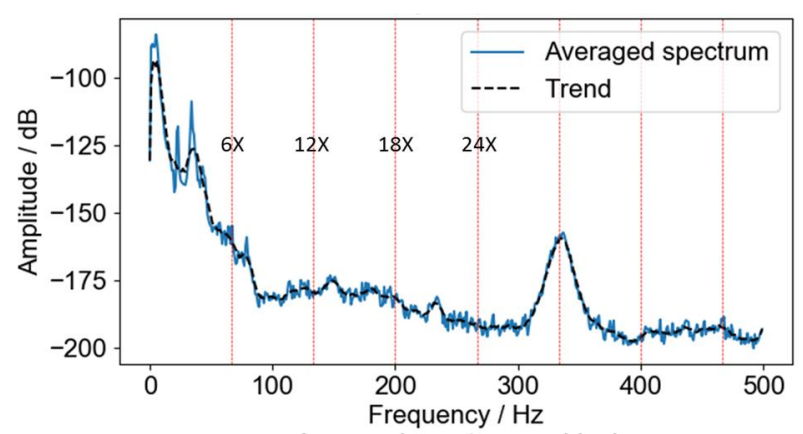

(a)

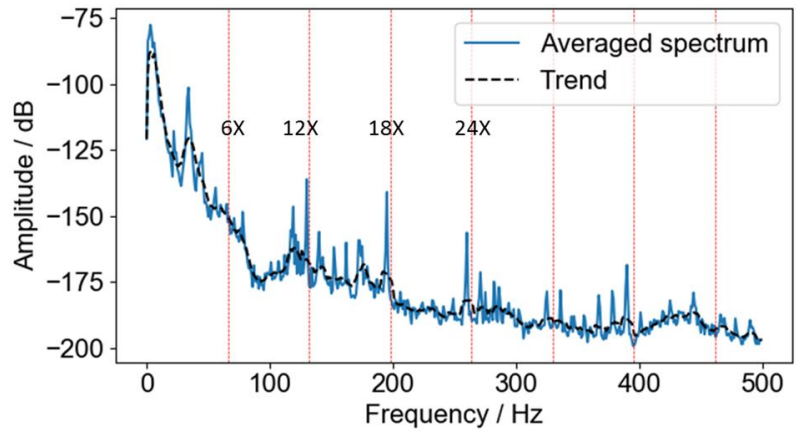

(b)

Figure 3 Averaged spectrum of the acoustic signals with (a) a healthy bearing, and (b) a faulty bearing

Although the averaged spectrum can differentiate the faulty bearing from the healthy one, there are still some limitations: (1) the amplitude of averaged spectrum may vary along with the microphone volume; (2) the amplitude of averaged spectrum may vary when vehicle driving speed changes. To remove the inconsistency, averaged spectrum residual is obtained to enhance the fault signature.

Firstly, the tendency curve is obtained by smoothing the averaged spectrum with a given smooth band width (we use $10 \mathrm{~Hz}$ by default), i.e. 10 -points moving average. And then the residual is obtained by subtracting the smoothed spectrum (tendency) from the averaged spectrum. By converting the averaged spectrum to the spectrum residual, the difference between the healthy bearing and the faulty bearing is enlarged, which helps to improve the diagnosis accuracy. Meanwhile, the values are normalized, which enhances the robustness and generalization of discovered fault signatures. Here the normalization is because the difference of two spectrums in log scale is equivalent to the division of two spectrum. That means the signal is normalized to its baseline amplitude. Two examples of averaged spectrum residual for a healthy bearing and a bad bearing are shown in Figure 4 (a) and (b), respectively. 


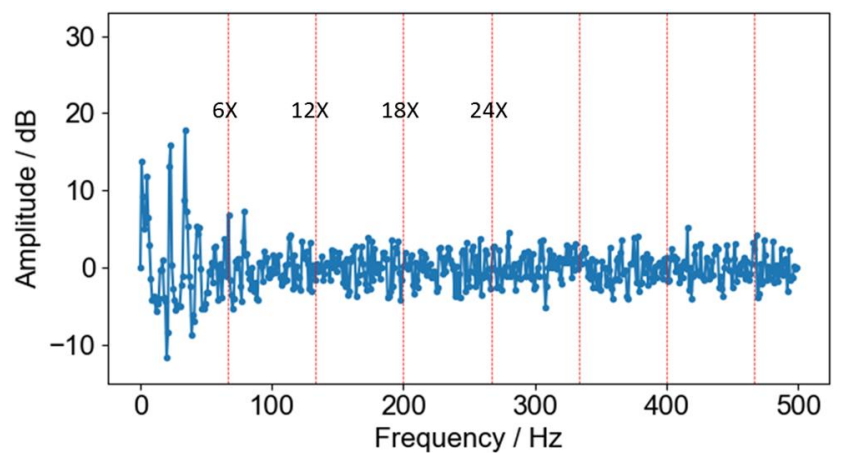

(a)

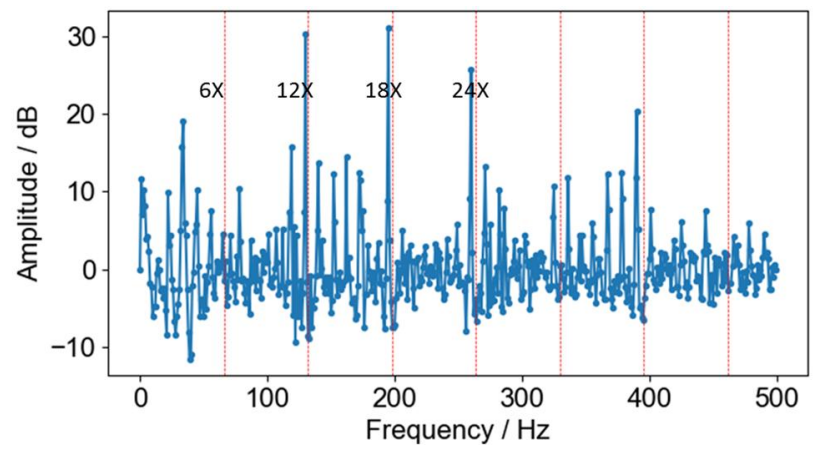

(b)

Figure 4 Averaged spectrum residual of acoustic signals with (a) a healthy bearing, and (b) a bad bearing

The harmonic amplitude at 12X, 18X, and $24 \mathrm{X}$ of fundamental frequency are extracted as features (i.e. fault indicators) to differentiate healthy bearings and faulty bearings. The features are defined as the max values in a narrow band centered at $12 \mathrm{X}, 18 \mathrm{X}$, and $24 \mathrm{X}$ fundamental frequencies. The band width is set as 1.6 times fundamental frequency by default. For example, if wheel speed is $10 \mathrm{~Hz}$, then features are the max values from following frequency area: $[120-8,120+8] \mathrm{Hz}, \quad[180-8,180+8] \mathrm{Hz}$, $[240-8,240+8] \mathrm{Hz}$. The bandwidth is determined by the accuracy of wheel speed estimation and the stability of the driving during the data collection. This value is adjustable based on the data property.

Table 1 lists the features extracted from several different test cases, e.g. 'Bad (30 mph)' means the test is performed with a bad bearing (e.g. degraded or faulty) in the cruise mode (vehicle speed is $30 \mathrm{mph}$ ). "F end" indicates the location of the microphone is front (on the dash board), and ' $R$ end' indicates the location of the microphone is in the rear trunk. In the bad cases listed below, the bad bearing is installed at the front left wheel. We can find that the feature values differ greatly between bad bearing and good bearing in $\mathrm{F}$ end.

The acoustic data from the healthy bearing and the bad bearing are used to select features. To inject the bearing fault, e.g. curb impact, the bearing is mounted to a rigid fixture with a moment arm bracket. A predefined force is applied to the
Table 1 Features extracted from different test cases

\begin{tabular}{|c|c|c|c|c|c|c|}
\hline \multirow{2}{*}{$\begin{array}{l}\text { Test } \\
\text { cases }\end{array}$} & \multicolumn{3}{|l|}{$\mathrm{F}$ end } & \multicolumn{3}{|l|}{$\mathrm{R}$ end } \\
\hline & $12 X$ & $18 \mathrm{X}$ & $24 X$ & $12 X$ & $18 \mathrm{X}$ & $24 X$ \\
\hline $\begin{array}{l}\text { Bad } \\
(30 \mathrm{mph})\end{array}$ & 24.40 & 23.58 & 26.67 & 16.70 & 8.21 & 3.18 \\
\hline $\begin{array}{l}\mathrm{Bad} \\
(50 \mathrm{mph})\end{array}$ & 26.06 & 30.68 & 23.70 & 22.89 & 13.03 & 4.42 \\
\hline $\begin{array}{l}\text { Bad } \\
(70 \mathrm{mph})\end{array}$ & 27.56 & 27.37 & 29.29 & 22.91 & 4.31 & 13.05 \\
\hline $\begin{array}{l}\text { Good } \\
\text { (30mph) }\end{array}$ & 6.68 & 10.12 & 1.53 & 8.82 & 9.63 & -0.75 \\
\hline $\begin{array}{l}\text { Good } \\
(50 \mathrm{mph})\end{array}$ & 5.53 & 2.67 & 3.43 & 2.24 & 2.99 & 3.14 \\
\hline
\end{tabular}

moment arm to damage the bearing. In this work, one healthy bearing and three damaged bearings with the impact force of $50 \mathrm{kN}, 60 \mathrm{kN}$, or $70 \mathrm{kN}$ are employed, respectively. The $50 \mathrm{kN}$ and $60 \mathrm{kN}$ bearings are considered as the degraded bearing and the $70 \mathrm{kN}$ bearing is considered as the bad bearing to be failed. The exact ratio of the Brinelling size to the roller element diameter is not available, since the bearing is sealed well and tested in the vehicle. However, during the vehicle test, the noise level introduced by the bearing is proportional to the impact force, which is evaluated by our service technician.

The acoustic data and wheel speed data are collected when different bearings are installed. Figure 5 shows the features extracted from the acoustic data with different bearings, where both 'healthy' and 'BentTieRod' are healthy bearings, 'bad_50kN' and 'bad_60kN' are degraded bearings, and 'bad_70kN' is the faulty bearing. From Figure 5, we can find out that the ' $12 \mathrm{X}$ harmonics' and the ' $18 \mathrm{X}$ harmonics' can separate the healthy bearing and faulty bearing very well with the largest distance between healthy data and faulty data, and the features in both groups are condensed. Compared with the feature ' $12 \mathrm{X}$ harmonics' and the feature ' $18 \mathrm{X}$ harmonics', the feature ' $24 \mathrm{X}$ harmonics' doesn't have a comparable performance. Therefore, the features ' $12 \mathrm{X}$ harmonics' and ' $18 \mathrm{X}$ harmonics' are selected as the fault signatures for bearing diagnosis.

In order to monitor and diagnose the wheel bearing fault onboard, a bearing fault diagnosis algorithm is triggered periodically. The algorithm processes the data acquired within given time span (e.g. 5 seconds), and makes a decision. We call this as 'single dataset wheel bearing FD module'. The flowchart is shown in Figure 6.

The input of the module is 4 wheels' speeds and the acoustic signal. When the data are imported into the module, the module parameters will be initialized. The module parameters include: time-frequency analysis parameters (window length, overlap ratio and interested frequency range) and the signal sampling rate. Thereafter, the spectrogram of the acoustic signals is calculated using STFT. To improve the spectrum analysis performance, the averaging and residual algorithms are executed. The features 


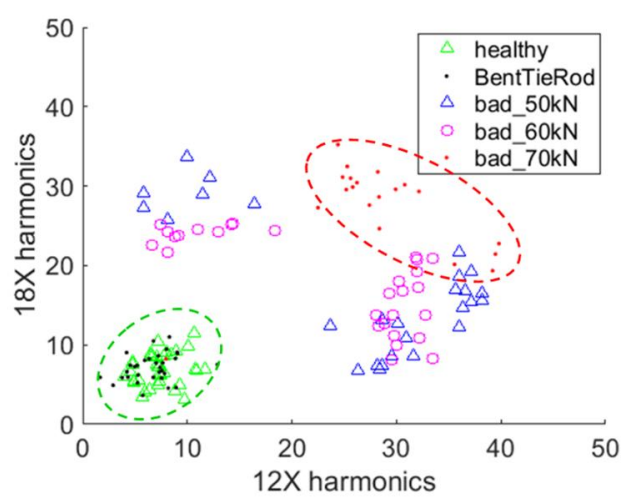

(a)

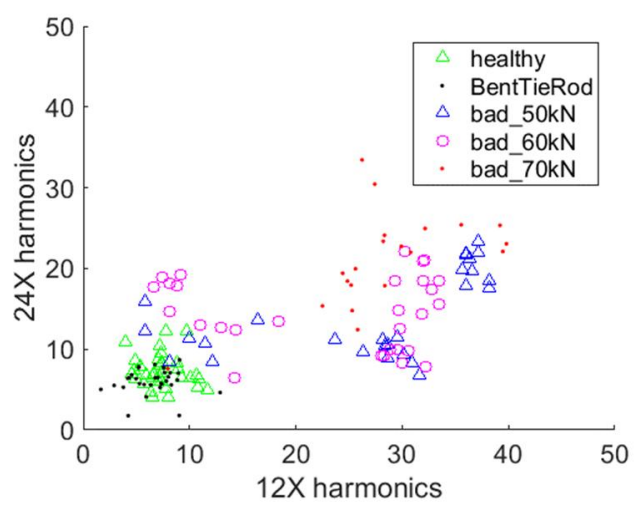

(b)

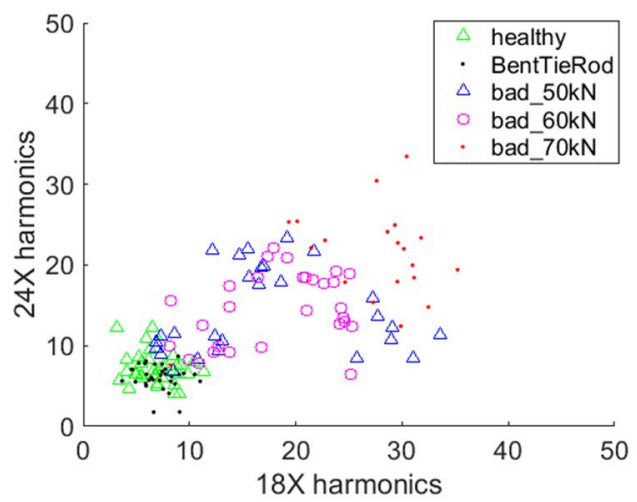

(c)

Figure 5 Feature visualization with different combinations: (a) $12 \mathrm{X}$ harmonics $+18 \mathrm{X}$ harmonics; (b) $12 \mathrm{X}$ harmonics + 24X harmonics; (c) $18 \mathrm{X}$ harmonics $+24 \mathrm{X}$ harmonics

are designed as the peaks at $12 \mathrm{X}$, and $18 \mathrm{X}$ fundamental frequency from residual spectrum. Finally, the diagnosis result is determined using a rule check. The rules involve 4 parameters $T 1, T 2, T 3$ and $T 4$ to differentiate the health condition for wheel bearings. Here T1 is the upper limit of Feature 1 to determine whether bearing is healthy or not, T2 is the upper limit of Feature 2 to determine whether bearing is healthy or not, T3 is the lower limit of Feature 1 to determine whether bearing is faulty or not, and T4 is the lower limit of Feature 2 to determine whether bearing is faulty or not. As depicted in the flowchart above, by comparing the feature data with $T 1, T 2, T 3$ and $T 4$, the bearing diagnosis result is obtained.

Single dataset wheel bearing FD module algorithm processes the data when the vehicle is driven in the cruise mode. But in the actual driving, the driving speed may have a lot of different patterns. In order to apply the algorithm to real-time driving environment, some enabling conditions are needed to trigger the single dataset wheel bearing FD module. Also, to decrease the false positive rate, a voting mechanism is added to make sure the diagnosis result is a comprehensive one based on different speed levels and multiple decisions. The overall wheel bearing FD model flowchart is shown in Figure 7.

When the data comes in, the first step is to check if the wheel speeds are stable (in cruise mode) or not. Here, we treat speed as stable, when the wheel speed variation less than $\Delta(0.5 \mathrm{~Hz}$ by default). If the input data meets this enabling condition, then the single dataset wheel bearing FD module will be called, and a diagnosis result based on this dataset is obtained. The wheel speed is divided into 3 levels by default, and the speed level of current dataset will be recorded in the buffer together with the diagnosis result. The enabling wheel speed range $[V 1, V 2]$ is determined by the vehicle design, and suggested driving speed. For example, if we know a vehicle's suggested driving speed is $40-70 \mathrm{mph}$, then the corresponding wheel speed can be obtained.

The buffer size is 15 by default. When the buffer is full, the overall diagnosis result will be given based on the information in the buffer. If there are more than 10 out of all 15 diagnosis results are "faulty", and there are at least 2 wheel speed levels in the buffer, then the overall diagnosis result is "bad bearing". If there are more than 10 out of all 15 diagnosis results are "faulty" but all the diagnosis results are based on the datasets from same wheel speed level, then the speed level information will be saved for the rule check next time. Otherwise if there are more than 10 out of 15 diagnosis results are either "faulty" or "degraded", then the overall diagnosis result is "degraded bearing". If it is not detected as "bad" or "degraded", then the overall diagnosis result is "healthy bearing". Once the overall diagnosis result is obtained, the buffer will be reset.

\section{Validation of the Proposed Solution}

In the previous section, we have introduced the whole procedure for the wheel bearing FD. To verify the proposed method, some experimental data are collected from our test vehicle for the wheel bearings with different health conditions.

As described before, the bad bearings with impact force $50 \mathrm{kN}, 60 \mathrm{kN}$ and $70 \mathrm{kN}$ are used in this work. All the 
algorithms including the single dataset FD module algorithm and the overall wheel bearing FD algorithm is tested below.

Firstly, the performance test is performed based on the organized test data samples. There are 141 test data samples (5 seconds each) in total including "healthy bearing", "degraded bearing" and "faulty bearing". If only "healthy bearing" and "faulty bearing" are considered, the detection

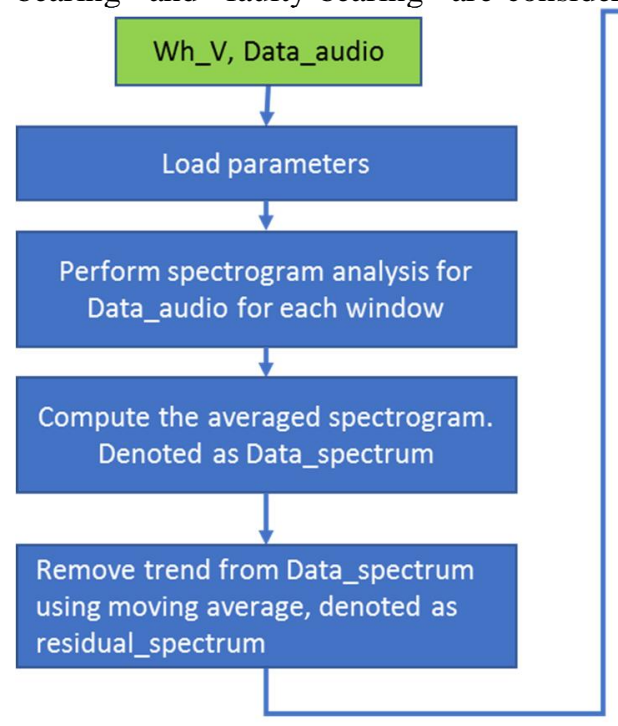

accuracy is $100 \%$ correct with 0 false positive rate. That's because the feature distribution between the healthy bearing and the faulty bearing are well separated. If we consider all

\section{Find max of residual_spectrum in the frequency band [12fr- bandwidth/2,12fr+bandwidth/2] and [18fr-bandwidth/2, $18 \mathrm{fr}+$ bandwidth/2], denoted as Feature1, Feature 2}

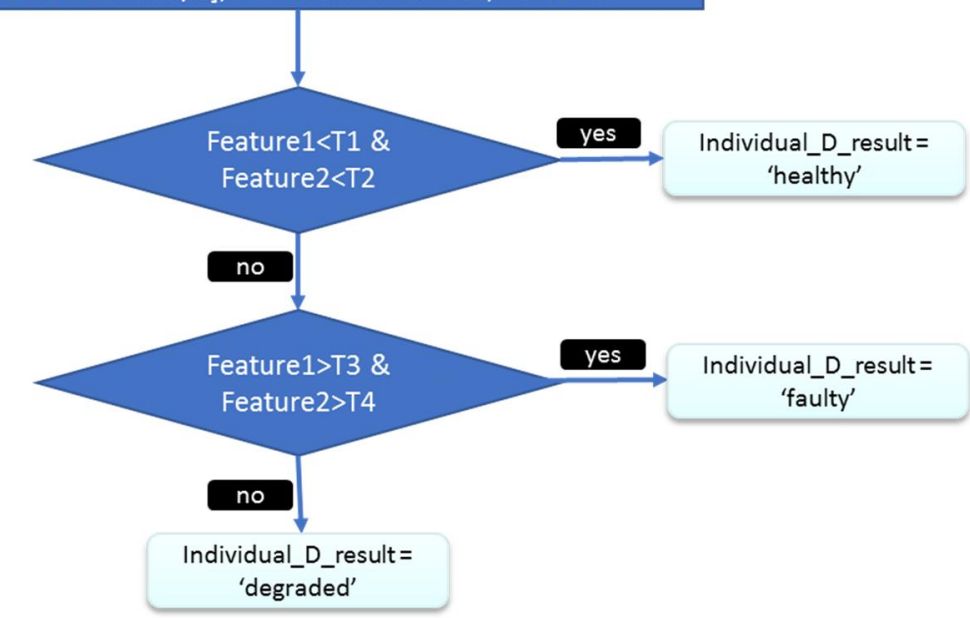

Figure 6. Single dataset wheel bearing FD module

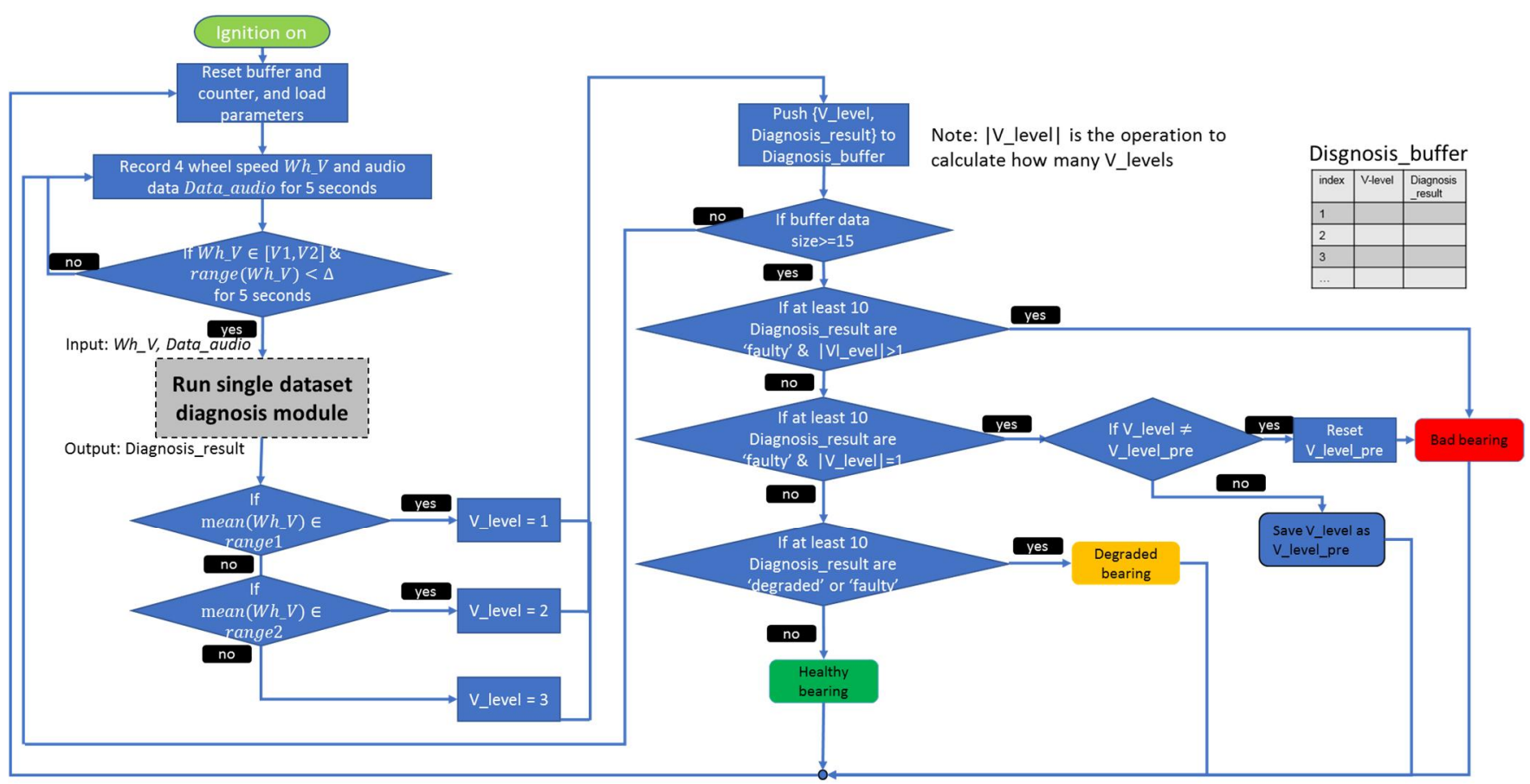

Figure 7. Flowchart of overall wheel bearing FD algorithm 
3 classes, the confusion matrix is shown in Figure 8. The detection accuracy of "healthy" is $100 \%$, and there are no "faulty" or "degraded" samples detected as "healthy", which means that the accuracy to separate healthy and degraded/faulty cases is $100 \%$. There are $4.84 \%$ "degraded" samples detected as "faulty". There are $31.58 \%$ "faulty" samples detected as "degraded". The performance for degraded and faulty cases indicates the need of decisionmaking logic to further improve the accuracy.

Secondly, some noisy data and various wheel speed data are used to further validate the robustness of the module. The first test is to check the algorithm on the acoustic data containing other noises. The test data contains different types of noises, such as driver talking, wind noise when window is down, radio playing and police siren. It is shown in Figure 9 that the single dataset bearing FD module is capable to detect the wheel bearing condition as healthy.

Considering that the wheel bearing FD will be deployed to the vehicle to realize real-time diagnosis, we evaluated the algorithm under several typical driving scenarios other than the cruise mode. As shown in Table 2, there are 4 test cases with different bearing conditions. Each test case includes several driving modes such as acceleration (acc), deceleration (dec), cruise, pass, stop sign and so on. The acoustic signals and wheel speed information are segmented to the 5-second data set and fed into the system continuously. Once 15 single data set results are generated, the overall diagnosis result will be given. To validate the bearing FD algorithm, the acoustic signal from the front microphone is used. For the first test case, all wheel bearings are healthy. Each single data set result in the buffer is healthy. The overall diagnosis result is "Good". For the second test case with a bad bearing $(70 \mathrm{kN})$, there are totally 30 single data set diagnostic results in the buffer, which are either "Faulty" or "Degraded". The overall

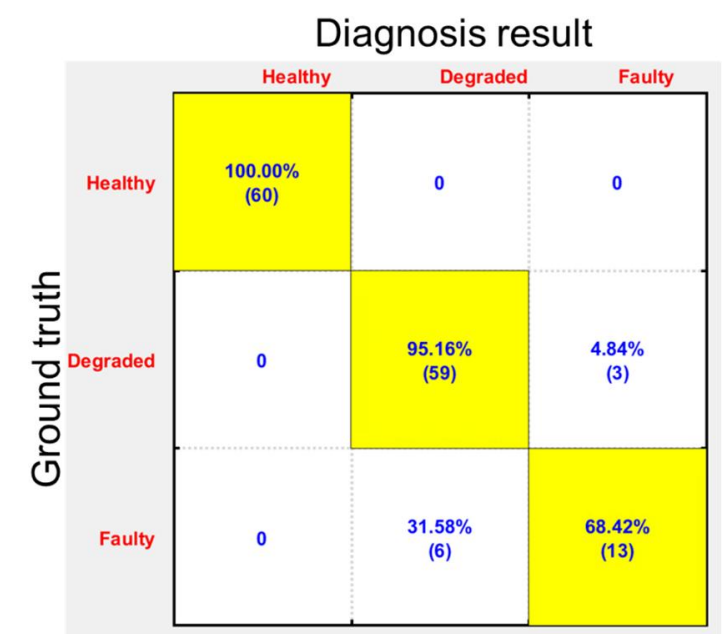

Figure 8. Confusion matrix of single dataset wheel bearing FD model

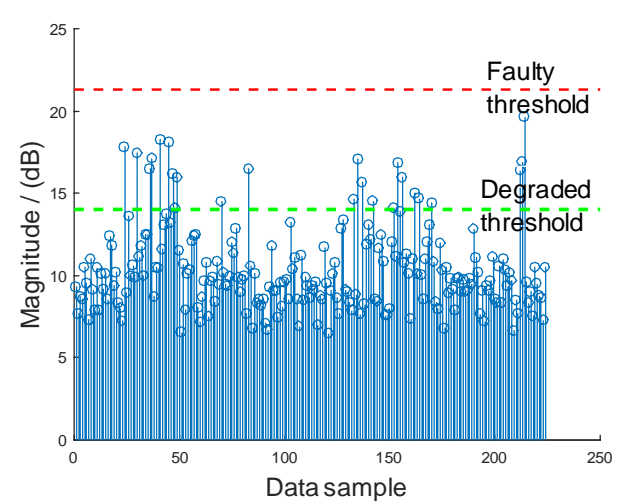

Figure 9. Max amplitude in the interested frequency band for the audio data

Table 2 Test cases of overall wheel bearing FD algorithm

\begin{tabular}{|c|c|c|c|}
\hline $\begin{array}{c}\text { Test } \\
\text { case }\end{array}$ & $\begin{array}{c}\text { Bearing } \\
\text { condition }\end{array}$ & Duration & Driving mode \\
\hline (a) & Healthy & $270 \mathrm{~s}$ & Acc, dec, cruise \\
\hline (b) & Faulty $(70 \mathrm{kN})$ & $700 \mathrm{~s}$ & $\begin{array}{c}\text { Cruise, stop, pass, } \\
\text { acc, dec }\end{array}$ \\
\hline (c) & $\begin{array}{c}\text { Degraded } \\
(60 \mathrm{kN})\end{array}$ & $420 \mathrm{~s}$ & Acc, dec, cruise, pass \\
\hline (d) & $\begin{array}{c}\text { Degraded } \\
(50 \mathrm{kN})\end{array}$ & $440 \mathrm{~s}$ & Acc, dec, cruise, pass \\
\hline
\end{tabular}

diagnosis result is "Bad". For the third and the forth test cases with degraded bearings, the overall diagnosis results are "degraded". The test results indicate the accuracy of the bearing FD algorithm is $100 \%$ without any false positive.

\section{Conclusion}

An acoustic-based approach is proposed and developed for vehicle wheel bearing fault detection and isolation in this work. It has been found that the wheel bearing fault can be diagnosed accurately using $12 \mathrm{X}$ and $18 \mathrm{X}$ wheel speed frequency response. The proposed fault diagnosis methods are robust to environmental noise, e.g. radio sound, driver talk, police siren and wind noise as well as different driving maneuvers, e.g. acceleration, deceleration, cruise, stop, and lane change. Even though the performance of the proposed approach is repeatable for different tests and different bearings, a physical explanation of the fault signature frequencies, i.e. $12 \mathrm{X}$ and $18 \mathrm{X}$ is still required. This will be out next focus along with the verification regarding to vehicle to vehicle variation and model to model variation.

\section{REFERENCES}

Jing, L., \& Qu, L. (2000). Feature extraction based on Morlet wavelet and its application for mechanical fault diagnosis. Journal of sound and vibration, vol. 234, pp. 135-148.

Kankar, P. K., Sharma, S. C., \& Harsha, S. P. (2011). Fault diagnosis of ball bearings using continuous wavelet 
transform. Applied Soft Computing, vol. 11, pp. 23002312.

Li, B., Chow, M.-Y., Tipsuwan, Y., \& Hung., J. C. (2000). Neural-network-based motor rolling bearing fault diagnosis. IEEE transactions on industrial electronics, vol. 47, pp. 1060-1069.

McInerny, S. A., \& Dai, Y. (2003). Basic vibration signal processing for bearing fault detection. IEEE Transactions on education, vol. 46, pp. 149-156.

Prieto, M. D., Cirrincione, G., Espinosa, A. G., Ortega, J. A., \& Henao., H. (2013). Bearing fault detection by a novel condition-monitoring scheme based on statistical-time features and neural networks. IEEE Transactions on Industrial Electronics, vol. 60, pp. 3398-3407.

Rai, V., \& Mohanty, A. (2007). Bearing fault diagnosis using FFT of intrinsic mode functions in Hilbert-Huang transform. Mechanical Systems and Signal Processing, vol. 21, pp. 2607-2615.

Randall, R. B., \& Antoni, J. (2011). Rolling element bearing diagnostics-A tutorial. Mechanical systems and signal processing, vol. 25, pp. 485-520.

Tan, C. C. (1990). Application of acoustic emission to the detection of bearing failures. International Tribology Conference 1990. Australia.

Yang, J., Zhang, Y., \& Zhu, Y. (2007). Intelligent fault diagnosis of rolling element bearing based on SVMs and fractal dimension. Mechanical Systems and Signal Processing, vol. 21, pp. 2012-2024.

Yu, D., Cheng, J., \& Yang, Y. (2005). Application of EMD method and Hilbert spectrum to the fault diagnosis of roller bearings. Mechanical systems and signal processing, vol. 19, pp. 259-270.

Zhang, H., Lu, S., He, Q., \& Kong, F. (2016). Multi-bearing defect detection with trackside acoustic signal based on a pseudo time-frequency analysis and Dopplerlet filter. Mechanical Systems and Signal Processing, vol. 70, pp. 176-200.

Zhang, Y., \& Du, X. (2014). Automatic field data analyzer for closed-loop vehicle design. Information Sciences, vol. 259, pp. 321-334.

\section{BIOGRAPHIES}

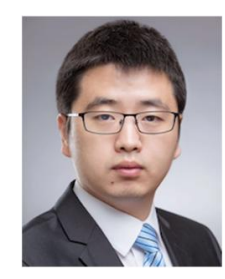

Jianshe Feng was born in Pingdingshan, China on June 24, 1990. He received the B.S. and M.S. in mechanical engineering from Tongji University, Shanghai, China in 2012 and Zhejiang University, Zhejiang, China in 2015, respectively. He is currently a Ph.D. researcher at Department of Mechanical
Engineering, University of Cincinnati, OH, USA. His research interests include prognostics and health management (PHM), machine learning and maintenance scheduling optimization with its industrial applications.

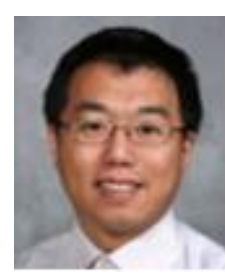

Xinyu Du received B.Sc. and M.Sc. degrees in automation from Tsinghua University, Beijing, China, in 2001 and 2004, respectively, and a Ph.D. in electrical engineering from Wayne State University, MI, USA, in 2012. He has been working at General Motors Global R\&D Center, Warren, MI, since 2010, and currently holds the staff researcher position in the vehicle systems research lab. His research interests include fuzzy hybrid system, vehicle health management, deep learning and data analytics. He has published more than 30 peer review papers and holds 33 patents or patent applications. He has been serving as an associate editor for Journal of Intelligent and Fuzzy Systems from 2012 and IEEE Access from 2018. He received the Boss Kettering Award from General Motors for his contribution in integrated starting system prognosis in 2015.

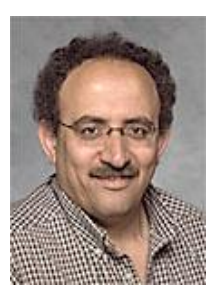

Mutasim Salman is currently working as a technical and management consultant specializing in Control Systems and Integrated Health Monitoring and Prognosis. He retired from General Motors Research, after 29 years of service where he was a Lab. Group Manager and a Senior Technical Fellow in the Electrical, Controls and Integration Lab. Dr. Salman received his bachelor's degree in Electrical Engineering from University of Texas at Austin; M.S. and $\mathrm{PhD}$ in Electrical Engineering with specialization in Systems and control from University of Illinois at UrbanaChampaign. He also has an Executive MBA. Dr. Salman has an extensive industrial and academic experience. His research focuses on modeling and simulation, diagnosis and control as well as energy management strategies development and validation for energy and automotive systems. Dr. Salman has done pioneering work in diagnosis and control for the automotive industry that led to the production of "Industry First" systems. He is the recipient of several awards that include 5 GM prestigious Boss Kettering, 3 McCuen, and 2 President and Chairman Awards. Dr. Salman holds 56 patents in diagnosis, control, and energy management of components and systems, 15 of them have already been used in products, and he has published more than 60 refereed technical papers and a chapter in a book on power systems modeling and simulation. 